\title{
Magneto-optical study of electron occupation and hole wave functions in stacked self-assembled InP quantum dots
}

\author{
M. Hayne, ${ }^{\text {a) }}$ J. Maes, and V. V. Moshchalkov \\ Laboratorium voor Vaste-Stoffysica en Magnetisme, Katholieke Universiteit Leuven, Celestijnenlaan 200D, \\ B-3001 Heverlee, Belgium \\ Y. M. Manz, O. G. Schmidt, and K. Eberl \\ Max-Planck-Institut für Festkörperforschung, Heisenbergstrasse 1, 70569 Stuttgart, Germany
}

(Received 20 November 2000; accepted for publication 14 May 2001)

\begin{abstract}
We have studied the magnetophotoluminescence of doubly stacked layers of self-assembled InP quantum dots in a GaInP matrix. 4.0 \pm 0.1 monolayers of InP were deposited in the lower layer of each sample, whereas in the upper layer 3.9, 3.4, and 3.0 monolayers were used. Low-temperature photoluminescence measurements in zero magnetic field are used to show that, in each case, only one layer of dots is occupied by an electron, and imply that when the amount of InP in both layers is the same, the dots in the upper layer are larger. High-field photoluminescence data reveal that the position and extent of the hole wave function are strongly dependent on the amount of InP in the stack. (C) 2001 American Institute of Physics. [DOI: 10.1063/1.1383807]
\end{abstract}

Self-assembled quantum dots offer the opportunity to study the effects of confinement in low-dimensional semiconductors and apply these findings to the development of advanced optoelectronic devices, such as lasers. ${ }^{1-3}$ InAs quantum dots in GaAs have applications in lasers for telecommunications, ${ }^{1}$ while red-light lasers ${ }^{2,3}$ based on InAlAs dots in AlGaAs (Ref. 2) or InP dots in GaInP (Ref. 3) could find applications in a number of sectors such as optical data storage digital video disk. In this letter, we discuss stacked layers of self-assembled InP dots in GaInP. We use photoluminescence spectroscopy in zero and high magnetic fields to address the basic problem of how and where the electrons and holes are confined within the sample, an issue which has clear relevance for both fundamental physics and applications, such as laser devices.

The physics of charge confinement in quantum dots can be approximated by a simple particle-in-a-box problem. In such a system, it is well known that the energy levels scale as $1 / L^{2}$, where $L$ is the size of the box. Furthermore, when the box is small, small changes to the dimensions have a large effect on the energy levels, but as the box gets larger changing its size has very little effect. This model can be very usefully applied to InP dots in GaInP, since as a Type II system, ${ }^{4,5}$ the electrons and holes can be considered to sit in different boxes. The electrons are well confined to the dots by a large conduction band offset. ${ }^{4}$ Our quantum dots are quite small and flat $(\sim 16 \mathrm{~nm}$ diameter and $2 \mathrm{~nm}$ height $){ }^{6}$ so the electron energy levels are very sensitive to the dot size. On the other hand, the application of a magnetic field, $B$, has a very small influence on such tightly confined charges. The reverse is true for the holes, which are weakly confined by strain $^{4}$ to the GaInP matrix between stacked layers of dots. ${ }^{5}$ As a result of this their wave function extent, $\sqrt{\left\langle\rho^{2}\right\rangle}$, is relatively large, and so they dominate the diamagnetic shift of the photoluminescence (PL) on the application of a strong

${ }^{a)}$ Electronic mail: manus.hayne@fys.kuleuven.ac.be magnetic field. For the same reason, the hole energy levels are rather insensitive to changes in $\sqrt{\left\langle\rho^{2}\right\rangle}$. Here, we exploit the role of size confinement effects by studying the zero and high-field PL of doubly stacked layers of vertically aligned self-assembled InP quantum dots in which the size of the dots in the upper layer is varied. We show that the zero-field PL is sensitive to the electron occupation of the dots, while the hole confinement can be determined by the high-field data. With this method, we can build a detailed picture of both electron and hole confinement in these structures which can then be used in the design of quantum-dot laser devices.

The samples were grown by solid-source molecularbeam epitaxy. ${ }^{2,6}$ Four $( \pm 0.1)$ monolayers $(\mathrm{ML})$ of InP were deposited on a GaInP buffer layer, which was grown on a GaAs substrate. After the deposition of a further $4 \mathrm{~nm}$ of GaInP, a second dot layer was grown with nominally 3.9, 3.4, and 3.0 ML of InP in samples A, B, and C, respectively. The distribution of the dot sizes in the samples is shown schematically in Fig. 1. PL measurements were taken at 4.2 $\mathrm{K}$ in pulsed magnetic fields up to $50 \mathrm{~T}$. Three spectra were taken during a typical $25 \mathrm{~ms}$ field pulse: one at the peak with a field variation of $\pm 0.1 \%$ over a $0.65 \mathrm{~ms}$ exposure time, and two during the down sweep with field resolutions and exposure times of $\pm 3 \%$ and $0.4-0.6 \mathrm{~ms}$, respectively. The sample was excited using a solid-state laser operating at 532 $\mathrm{nm}$ via a $400 \mu \mathrm{m}$ core optical fiber at a power density of about $1 \mathrm{~W} \mathrm{~cm}^{-2}$. The PL was collected by a bundle of six optical fibers surrounding the central laser fiber, and analyzed in a $0.25 \mathrm{~m}$ spectrometer with an intensified charge coupled device detector. The center of mass of the peak (peak position) was determined by numerical analysis of the PL data.

The zero-field spectra for the three samples ( $8 \mathrm{~K}$ data) are shown in Fig. 1. In each case, the peak at $1.75 \mathrm{eV}$ corresponds to the recombination from the InP dots, whereas the peak at about $1.95 \mathrm{eV}$ is from the GaInP matrix. The separation of the peaks demonstrates the strong confinement (of 


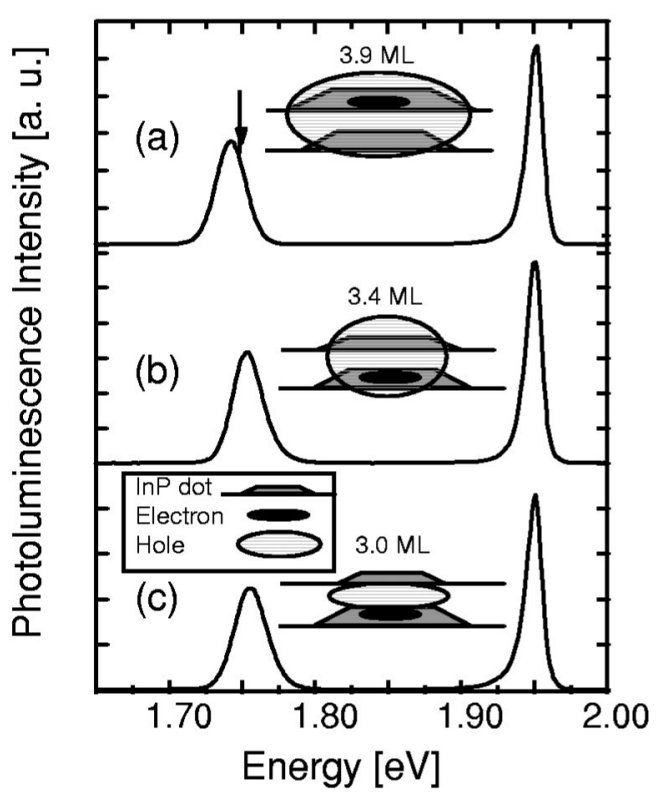

FIG. 1. Zero-field PL spectra for (a) sample A, (b) sample B, and (c) sample $\mathrm{C}$ taken at $8 \mathrm{~K}$ are shown. In each case, the high-energy peak is from the GaInP and the low-energy peak is from the dots. The arrow in the upper panel indicates the peak position of a single layer (4.0 ML) sample. The schematic drawings show the sample structure, the electron occupancy, and the hole wave function distribution inferred from our data in each case.

the electrons) in this system. It can be seen that the PL from the dots in each of the samples is very similar, in particular, the linewidth is rather narrow and shows very little variation between the samples (Table I). At first, this is somewhat surprising. Sample A has the same amount of InP in each layer, so one might expect the dots to be the same size and emitting light over the same range of energies. However, in samples B and C, there is a very significant difference in the amount of InP in the two layers, $33 \%$ in the case of sample C. Despite this, the PL line remains narrow. This can only be explained if just one of the layers of dots is contributing to the PL, i.e., only one layer of dots is occupied by electrons. Since the dots in the lower layers of samples B and C are larger than those in the upper layer and so have lower energy levels, we would expect that it is these which are occupied. A closer examination of the data confirms this assertion. The values in Table I show that the zero-field PL spectra from samples B and C are essentially identical. Since the size of the dots in the upper layer is different for these two samples, we can conclude that the electrons do indeed occupy the lower layer of dots. Further confirmation of this is found by comparing the position of the quantum-dot PL peak in these samples to that of a sample in which only a single layer of 4.0 ML dots is grown, as indicated by the arrow in the upper

TABLE I. Summary of the results of the zero-field and in-field PL experiments $B \| z$ is shown. With $B \perp z, \mu$ and $\sqrt{\left\langle\rho^{2}\right\rangle}$ were found to $0.27 m_{0}$ and 7.5 $\mathrm{nm}$, respectively for sample B.

\begin{tabular}{lccccc}
\hline \hline $\begin{array}{l}\text { Sample, InP in } \\
\text { upper layer } \\
\text { (ML) }\end{array}$ & $\begin{array}{c}\text { PL peak } \\
\text { position at } B=0 \\
(\mathrm{eV})\end{array}$ & $\begin{array}{c}\text { PL } \\
\text { linewidth } \\
(\mathrm{meV})\end{array}$ & $\begin{array}{c}\mu \\
\left(m_{0}\right)\end{array}$ & $\begin{array}{c}\sqrt{\left\langle\rho^{2}\right\rangle} \\
(\mathrm{nm})\end{array}$ & $\begin{array}{c}\text { Shift of PL } \\
\text { peak }(B \perp z) \\
(\mathrm{meV})\end{array}$ \\
\hline $\mathrm{A}, 3.9$ & 1.738 & 24 & 0.14 & 9.6 & 9.7 \\
$\mathrm{~B}, 3.4$ & 1.748 & 26 & 0.16 & 7.6 & 8.2 \\
$\mathrm{C}, 3.0$ & 1.750 & 27 & 0.20 & 7.3 & 5.4
\end{tabular}

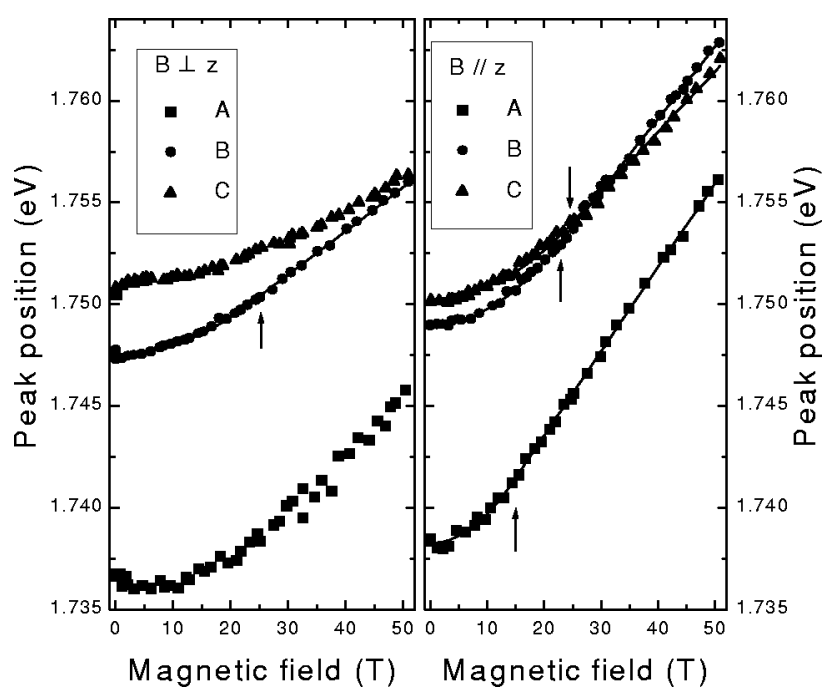

FIG. 2. PL peak position for the three samples with (a) $B \perp z$ and (b) $B \| z$ is shown. The arrows indicate the point at which the curve becomes linear, i.e., goes from the low-field to the high-field regime. The small difference in $B$ $=0$ energies for the same sample in different orientations are due to changes in the position of the laser spot on the sample.

panel of Fig. 1. The energy of the PL corresponds exactly with that of samples B and C.

We now turn to sample A. In this sample, the PL line is slightly narrower, and the line is at $10 \mathrm{meV}$ lower energy. Both these facts imply the presence of larger dots. Since we know that the dots in the lower layers of each of the samples are the same size, we conclude that in sample A the dots in the upper layer are larger. Both layers contain the same amount of InP, so we attribute the existence of larger dots in the upper layer to their more rapid nucleation as part of the stacking process, with a corresponding reduction in the thickness of the wetting layer. Such an effect has previously been directly observed in transmission electron microscopy measurements of stacked layers of $\mathrm{Ge}$ dots in $\mathrm{Si}^{7}$ Before going on to discuss the high-field data, we note that a strong occupation of only one dot in the stack requires efficient tunneling of the electrons between the dots. Such tunneling was recently observed in time-resolved PL measurements on triply stacked layers of self-assembled InP quantum dots, ${ }^{8}$ similar to those we studied previously.,

The dependence of the peak position of the quantum-dot (PL) line on the magnetic field for the three samples is shown in Fig. 2. We first discuss the data with the field applied perpendicular $(\perp)$ to the growth direction $(z)$, as shown in Fig. 2(a). In sample B, the peak shows conventional parabolic and linear field dependence, ${ }^{5,9}$ and we find values for the effective mass, $\mu$, and $\sqrt{\left\langle\rho^{2}\right\rangle}$ of $0.27 m_{0}$ and $7.5 \mathrm{~nm}$, respectively. The large $\mu$ confirms that the shift of the PL peak is determined by the holes, ${ }^{6}$ while $\sqrt{\left\langle\rho^{2}\right\rangle}$ $=7.5 \mathrm{~nm}$ indicates that in this sample, the holes extend vertically through the stack. Such a quantitative analysis is not possible for the other two samples since the shift of the PL line deviates from conventional behavior. An even more complicated dependence of the PL energies on $B \perp z$ was observed for triply stacked dots, ${ }^{9}$ but is yet to be explained. However, useful information can be obtained from a simple comparison of the total shift of the PL line for the three sets 
of data (last column of Table I). Here, we see a systematic reduction in the size of the shift as the amount of InP in the upper layer of dots is decreased, indicative of a reduction in the extent of the hole wave function. In particular, we note that in sample $\mathrm{C}$ the shift is $5.4 \mathrm{meV}$, a typical value for the case when the holes are confined entirely to the GaInP matrix between the stacked dots. ${ }^{5}$

The PL data with $B$ applied parallel (II) to $z$ confirms this picture. In the first instance, we are able to determine $\mu$ and $\sqrt{\left\langle\rho^{2}\right\rangle}$ in all three samples for this orientation (Table I). The masses are lower than for sample $\mathrm{B}$ with the $B \perp z$, but still considerably larger than the bulk InP exciton mass $\left(0.0678 m_{0}\right)$. This again confirms that the effect of the applied field is on the hole energy. It can also be seen that the hole masses in samples A and B are similar, but in sample C it is $30 \%$ larger. This is a direct result of the extent of the hole wave function vertically through the stack. The exciton effective mass in GaInP is known to be much higher than in InP. ${ }^{10}$ Thus, when the holes extend vertically through the entire stack and sample both the InP and the GaInP material, we expect them to have a lower mass than when they are confined entirely within the GaInP. Very similar mass values were found for triply stacked layers when the holes were confined to the GaInP $\left(\sim 0.23 m_{0}\right)$ between triply stacked dots, and when two such regions were coupled by the holes via the intervening InP dot $\left(\sim 0.16 m_{0}\right) .{ }^{5}$ The effective mass in the plane of the dots is a probe of the environment of the hole, and thus also depends on the material parameters which the hole wave function samples due to its extension in the growth direction. The conclusion that the holes extend vertically through the stack in samples A and B but not in sample $\mathrm{C}$ is consistent with our data with $B \perp z$. Finally, we discuss $\sqrt{\left\langle\rho^{2}\right\rangle}$ in the plane of the layers. A systematic shrinkage is seen as the amount of InP decreases. This is a result of the reduction in the volume of the strain field, which strongly determines the extent of the holes. Combining all of this information about the holes, we can draw what should be quite realistic representations of the hole wave functions in these samples. These, along with electron occupancies, are also shown in Fig. 1.

In conclusion, we have shown that the zero-field PL peak position is a sensitive probe of electron levels in InP quantum dots, and have used this to determine the electron occupancy in samples where the size of the dots in the upper of two layers is varied. High-field measurements have been shown to be sensitive only to the hole wave functions, and have allowed us to obtain their form and location in these samples.

This work was supported by the FWO-Vlaanderen, the Flemish GOA, the Belgian IUAP programmes, and the Flemish Institute for the Promotion of Scientific-Technological Research in Industry (IWT). One of the authors (M.H.) is a fellow of the IWT.

${ }^{1}$ For a recent review see: D. Bimberg, M. Grundmann, F. Heinrichsdorf, N. N. Ledenstov, V. M. Ustinov, A. E. Zhukov, A. R. Kovsh, M. V. Maximov, Y. M. Shernyakov, B. V. Volovik, A. F. Tsatsul'nikov, P. S. Kop'ev, and Z. I. Alferov, Thin Solid Films 367, 235 (2000).

${ }^{2}$ Y. M. Manz, O. G. Schmidt, and K. Eberl, Appl. Phys. Lett. 76, 3343 (2000).

${ }^{3}$ K. Hinzer, J. Lapointe, Y. Feng, A. Delâge, S. Fafard, A. J. SpringThorpe, and E. M. Griswold, J. Appl. Phys. 87, 1496 (2000).

${ }^{4}$ C. Pryor, M.-E. Pistol, and L. Samuelson, Phys. Rev. B 56, 10404 (1997).

${ }^{5}$ M. Hayne, R. Provoost, M. K. Zundel, Y. M. Manz, K. Eberl, and V. V. Moshchalkov, Phys. Rev. B 62, 10324 (2000).

${ }^{6}$ M. K. Zundel, P. Specht, K. Eberl, N. Y. Jin-Phillipp, and F. Phillipp, Appl. Phys. Lett. 71, 2972 (1997).

${ }^{7}$ O. G. Schmidt, O. Kienzle, Y. Hao, and K. Eberl, Appl. Phys. Lett. 74, 1272 (1999); O. Kienzle, F. Ernst, M. Rühle, O. G. Schmidt, and K. Eberl, ibid. 74, 269 (1999).

${ }^{8}$ A. Christ, H. Giessen, W. W. Rühle, K. Korona, J. Kuhl, M. Zundel, Y. M. Manz, and K. Eberl, Phys. Status Solidi B 221, 59 (2000).

${ }^{9}$ R. Provoost, M. Hayne, V. V. Moshchalkov, M. K. Zundel, and K. Eberl, Appl. Phys. Lett. 75, 799 (1999).

${ }^{10}$ P. Ernst, Yong Zang, F. A. J. M. Driessen, A. Mascarenhas, E. D. Jones, G. Geng, F. Scholz, and H. Schweizer, J. Appl. Phys. 81, 2814 (1997). 